\title{
$\Phi$-measure of pions in high energy heavy-ion collisions
}

\author{
Q. H. Zhang \\ Institute of Theoretical Science and Department of Physics, University of Oregon, Eugene, \\ Orgeon 97403-5203 \\ Physics Department, McGill University, Montreal QC H3A 2T8, Canada
}

\begin{abstract}
Event-by-Event fluctuations for high energy heavy-ion collisions are investigated. The $\Phi$-measures are calculated for pions. It is found that multiparticle symmetrization correlation has great influence on the value of $\Phi$ if the phase space density is high. Previous derivation of $\Phi$-measure has neglected multiparticle quantum correlation. So the explanation of $\Phi$-measure as a measure of dynamical correlation is reasonable only when multiparticles quantum correlation effects are smaller. We argue that the $\Phi$-measure might be applicable at the SPS energy where the pion phase space density is much less than one.
\end{abstract}

\section{INTRODUCTION}

One of the main goals of relativistic heavy-ion physics is the discovery of the new state of strongly interacting matter that is called the quark-gluon plasma(QGP) [1]. As one can not detect QGP directly, lots of indirect signals are suggested [1]. It has been pointed out that the study of event-by-event fluctuations in high energy heavy-ion collisions may provide us more information about the experiment [2 [5]. For examples, event-by-event fluctuations may provide us information about the heat capacity [ [, 6, 8], possible equilibration of the system [9 17] or about the phase transition [18,8]. Experimental group NA49 has already studied the $\Phi$-measure in $\mathrm{Pb}+\mathrm{Pb}$ collisions at SPS energy [19]. Recently Bialas, Koch [20] and Belkacem et. al. [21] have found a relationship between the moments of event-by-event fluctuations and the inclusive correlations. In this paper we will extend the derivation given in [21], and connect the result of Ref. [20] with Ref. [21]. It has been suggested in Ref. [9] that if there is no special dynamics involved in nuclei and nuclei $(A A)$ collisions, then the $\Phi$ measure for $A A$ should be the same as the $\Phi$-measure for proton and proton ( $p p)$ collisions. So by comparing $\Phi$-measures for pp and AA, one can get information on the dynamics that is involved in $A A$ collisions. However, the derivation in Ref. [9] has neglected the multiparticles Bose-Einstein symmetrization effects among the identical particles. The difference between $\Phi$-measure for $A A$ and that for $p p$ collisions may be due to the multi-particles BoseEinstein correlation so that the statement of Ref. [9] given above is no longer valid anymore. In Ref. [12], the author has assumed that the pions produced from $A A$ collisions has a BoseEinstein distribution form in momentum space and has used this spectrum distribution to calculate $\Phi$-measure and found that $\Phi$-measure of Bose-Einstein symmetrization is around the prediction of the experiment. But it has been shown in Ref. [22] that only when the 
volume goes to infinity, the spectrum distribution of pions can be of the function form of Bose-Einstein. In this paper, we will calculate the $\Phi$-measure of pions in heavy-ion collisions by using a pion distribution function in both coordinate and momentum space. As has been shown in Ref. [23 that when coordinate space volume goes to infinity, multi-particle BoseEinstein correlation will make the pion spectrum distribution change to Bose-Einstein form in momentum space. So the calculation in Ref. [12] can be achieved in our calculation by letting the volume goes to infinity. On the other side, the effects of the finite size of $A A$ system on $\Phi$-measure can be studied in our calculation. This paper is arranged in the following way: In section II, the relationship between $\Phi$-measure and particles inclusive distributions are derived. In section III, we first derive the formulas which will be used for the calculation of $\Phi$-measure of pions, then we calculate the $\Phi$-measure for different system size and temperature. For comparison, the $\Phi$-measure of pions based on the method given in Ref. 12 are also calculated. In section IV, a simple model is given which demonstrates explicitly that the derivation given in Ref. [17] has neglected multi-particles Bose-Einstein symmetrization effects and conclusions given in Ref. [17] only holds when the multi-particles symmetrization effects are smaller. Finally, Conclusions are given in Section V.

\section{THE RELATIONSHIP BETWEEN $\Phi$-MEASURE AND PARTICLES INCLUSIVE DISTRIBUTION}

The quantity $Z(y)$ for an arbitrary event is defined as [9, 20]:

$$
Z(y)=\sum_{i=1}^{N} y\left(\mathbf{p}_{\mathbf{i}}\right),
$$

here $N$ is the multiplicity of the event and $y\left(\mathbf{p}_{\mathbf{i}}\right)$ is the function of momentum $\mathbf{p}_{\mathbf{i}}$ in the event 1

Then the k-th order moments of $Z$ can be written down as [20,21]

\footnotetext{
${ }^{1}$ If $y\left(\mathbf{p}_{\mathbf{i}}\right)$ is total momentum, $y\left(\mathbf{p}_{\mathbf{i}}\right)=\left|p_{i}\right|$. If $y\left(\mathbf{p}_{\mathbf{i}}\right)$ is transverse momentum, $y\left(\mathbf{p}_{\mathbf{i}}\right)=\sqrt{p_{1}^{2}+p_{2}^{2}}$. If $y\left(\mathbf{p}_{\mathbf{i}}\right)$ is the rapidity, then we need to know the energy of particles $i$. If $y\left(\mathbf{p}_{\mathbf{i}}\right)$ is electric charge of the particles, we also need to know the electrical charge of particles $i$. So Eq.(1) can be generalized to

$$
Z(y)=\sum_{i=1}^{N} y\left(\mathbf{p}_{\mathbf{i}}, s_{i}\right),
$$

Here $s_{i}$ represents other property of particles $i$, like mass and charge. One can simply write Eq.(2) as

$$
Z(y)=\sum_{i=1}^{N} y_{i}
$$

Here $y_{i}$ is the function of $i$-th particle. 


$$
\left\langle Z(y)^{k}\right\rangle=\frac{1}{M} \sum_{j=1}^{M}\left[\sum_{i=1}^{N_{j}} y\left(\mathbf{p}_{\mathbf{i}}\right)\right]^{k},
$$

where $M$ is the total number of events and $N_{j}$ is the multiplicity of the $j$-th event.

In the following we will use $N$-body distribution function $f\left(N, \mathbf{p}_{\mathbf{1}}, \cdots, \mathbf{p}_{\mathbf{N}}\right)$ which is normalized as

$$
\int d \mathbf{p}_{\mathbf{1}} \cdots d \mathbf{p}_{\mathbf{N}} f\left(N, \mathbf{p}_{\mathbf{1}}, \cdots, \mathbf{p}_{\mathbf{N}}\right)=P(N) .
$$

Here $P(N)$ is the normalized multiplicity distribution.

Following Ref. [21], we define the reduced s-body distribution functions for a system of indistinguishable $N$-particles as:

$$
f_{s}\left(N, \mathbf{p}_{\mathbf{1}}, \ldots, \mathbf{p}_{\mathbf{s}}\right)= \begin{cases}\frac{N !}{(N-s) !} \int \mathbf{p}_{\mathbf{s}+\mathbf{1}} \cdots d \mathbf{p}_{\mathbf{N}} f\left(N, \mathbf{p}_{\mathbf{1}}, \cdots, \mathbf{p}_{\mathbf{N}}\right) & s<N \\ N ! f\left(N, \mathbf{p}_{\mathbf{1}}, \cdots, \mathbf{p}_{\mathbf{N}}\right) & s=N \\ 0 & s>N\end{cases}
$$

But we should point out that this definition is different from that in Ref. [21] ]

According to Eq.(4), One can write out the $k$-order moments of $Z$ as 20,21]:

$$
\left\langle Z(y)^{k}\right\rangle=\sum_{N=0}^{\infty} \int d \mathbf{p}_{\mathbf{1}} \cdots d \mathbf{p}_{\mathbf{N}}\left[\sum_{i=1}^{N} y\left(\mathbf{p}_{\mathbf{i}}\right)\right]^{k} f\left(N, \mathbf{p}_{\mathbf{1}}, \cdots, \mathbf{p}_{\mathbf{N}}\right) .
$$

To be clear, the first to fifth order moments of $\mathrm{Z}$ can be expressed as:

$$
\begin{gathered}
\langle Z(y)\rangle=\sum_{N=0}^{\infty} \int d \mathbf{p}_{\mathbf{1}} y\left(\mathbf{p}_{\mathbf{1}}\right) f_{1}\left(N, \mathbf{p}_{\mathbf{1}}\right), \\
\left\langle Z(y)^{2}\right\rangle=\sum_{N=0}^{\infty}\left[\int d \mathbf{p}_{\mathbf{1}} y^{2}\left(\mathbf{p}_{\mathbf{1}}\right) f_{1}\left(N, \mathbf{p}_{\mathbf{1}}\right)+\int d \mathbf{p}_{\mathbf{1}} d \mathbf{p}_{\mathbf{2}} y\left(\mathbf{p}_{\mathbf{1}}\right) y\left(\mathbf{p}_{\mathbf{2}}\right) f_{2}\left(N, \mathbf{p}_{\mathbf{1}}, \mathbf{p}_{\mathbf{2}}\right)\right], \\
\left\langle Z(y)^{3}\right\rangle=\sum_{N=0}^{\infty}\left[\int d \mathbf{p}_{\mathbf{1}} y^{3}\left(\mathbf{p}_{\mathbf{1}}\right) f_{1}\left(N, \mathbf{p}_{\mathbf{1}}\right)+3 \int d \mathbf{p}_{\mathbf{1}} d \mathbf{p}_{\mathbf{2}} y^{2}\left(\mathbf{p}_{\mathbf{1}}\right) y\left(\mathbf{p}_{\mathbf{2}}\right) f_{2}\left(N, \mathbf{p}_{\mathbf{1}}, \mathbf{p}_{\mathbf{2}}\right)\right. \\
\left.+\int d \mathbf{p}_{\mathbf{1}} d \mathbf{p}_{\mathbf{2}} d \mathbf{p}_{\mathbf{3}} y\left(\mathbf{p}_{\mathbf{1}}\right) y\left(\mathbf{p}_{\mathbf{2}}\right) y\left(\mathbf{p}_{\mathbf{3}}\right) f_{3}\left(N, \mathbf{p}_{\mathbf{1}}, \mathbf{p}_{\mathbf{2}}, \mathbf{p}_{\mathbf{3}}\right)\right],
\end{gathered}
$$

${ }^{2}$ In Ref. [21], the authors have defined a function $f_{s}\left(N, \mathbf{p}_{\mathbf{1}}, \ldots, \mathbf{p}_{\mathbf{s}}\right)$ as

$$
f_{s}\left(N, \mathbf{p}_{\mathbf{1}}, \ldots, \mathbf{p}_{\mathbf{s}}\right)=\frac{N !}{(N-s) !} \int d \mathbf{p}_{\mathbf{s}+\mathbf{1}} \ldots d \mathbf{p}_{\mathbf{N}} f_{N}\left(N, \mathbf{p}_{\mathbf{1}}, \ldots \mathbf{p}_{\mathbf{N}}\right) .
$$

It is clear that when $s=N$, the above formula is "bad" defined while this situation does not exist in our definition (see Eq.(6)). On the other hand, one can derive from Eq.(6) of this paper that

$$
f_{s}\left(N, \mathbf{p}_{\mathbf{1}}, \ldots, \mathbf{p}_{\mathbf{s}}\right)=\frac{1}{(N-s) !} \int d \mathbf{p}_{\mathbf{s}+\mathbf{1}} \ldots d \mathbf{p}_{\mathbf{N}} f_{N}\left(N, \mathbf{p}_{\mathbf{1}}, \ldots \mathbf{p}_{\mathbf{N}}\right) .
$$

From Eq.(7) and Eq.(8), we know that this two definitions (Ref. 21] and here) are different. 


$$
\begin{aligned}
\left\langle Z(y)^{4}\right\rangle= & \sum_{N=0}^{\infty}\left[\int d \mathbf{p}_{1} y^{4}\left(\mathbf{p}_{1}\right) f_{1}\left(N, \mathbf{p}_{1}\right)+4 \int d \mathbf{p}_{1} d \mathbf{p}_{2} y^{3}\left(\mathbf{p}_{1}\right) y\left(\mathbf{p}_{2}\right) f_{2}\left(N, \mathbf{p}_{1}, \mathbf{p}_{2}\right)\right. \\
& +3 \int d \mathbf{p}_{1} d \mathbf{p}_{2} y^{2}\left(\mathbf{p}_{1}\right) y^{2}\left(\mathbf{p}_{2}\right) f_{2}\left(N, \mathbf{p}_{1}, \mathbf{p}_{2}\right) \\
& +6 \int d \mathbf{p}_{1} d \mathbf{p}_{2} d \mathbf{p}_{3} y^{2}\left(\mathbf{p}_{\mathbf{1}}\right) y\left(\mathbf{p}_{\mathbf{2}}\right) y\left(\mathbf{p}_{\mathbf{3}}\right) f_{3}\left(N, \mathbf{p}_{1}, \mathbf{p}_{2}, \mathbf{p}_{3}\right) \\
& \left.+\int d \mathbf{p}_{1} d \mathbf{p}_{2} d \mathbf{p}_{3} d \mathbf{p}_{4} y\left(\mathbf{p}_{1}\right) y\left(\mathbf{p}_{2}\right) y\left(\mathbf{p}_{3}\right) y\left(\mathbf{p}_{4}\right) f_{4}\left(N, \mathbf{p}_{\mathbf{1}}, \mathbf{p}_{\mathbf{2}}, \mathbf{p}_{\mathbf{3}}, \mathbf{p}_{\mathbf{4}}\right)\right] \\
\left\langle Z(y)^{5}\right\rangle= & \sum_{N=0}^{\infty}\left[\int d \mathbf{p}_{1} y^{5}\left(\mathbf{p}_{1}\right) f_{1}\left(N, \mathbf{p}_{1}\right)+5 \int d \mathbf{p}_{1} d \mathbf{p}_{2} y^{4}\left(\mathbf{p}_{1}\right) y\left(\mathbf{p}_{\mathbf{2}}\right) f_{2}\left(N, \mathbf{p}_{\mathbf{1}}, \mathbf{p}_{\mathbf{2}}\right)\right. \\
+ & 10 \int d \mathbf{p}_{1} d \mathbf{p}_{\mathbf{2}} y^{3}\left(\mathbf{p}_{1}\right) y^{2}\left(\mathbf{p}_{2}\right) f_{2}\left(N, \mathbf{p}_{1}, \mathbf{p}_{2}\right) \\
+ & 10 \int d \mathbf{p}_{1} d \mathbf{p}_{2} d \mathbf{p}_{3} y^{3}\left(\mathbf{p}_{1}\right) y\left(\mathbf{p}_{2}\right) y\left(\mathbf{p}_{3}\right) f_{3}\left(N, \mathbf{p}_{1}, \mathbf{p}_{2}, \mathbf{p}_{3}\right) \\
+ & 15 \int d \mathbf{p}_{1} d \mathbf{p}_{2} d \mathbf{p}_{3} y^{2}\left(\mathbf{p}_{1}\right) y^{2}\left(\mathbf{p}_{2}\right) y\left(\mathbf{p}_{3}\right) f_{3}\left(N, \mathbf{p}_{1}, \mathbf{p}_{2}, \mathbf{p}_{3}\right) \\
+ & 10 \int d \mathbf{p}_{1} d \mathbf{p}_{2} d \mathbf{p}_{3} y^{2}\left(\mathbf{p}_{1}\right) y\left(\mathbf{p}_{2}\right) y\left(\mathbf{p}_{3}\right) y\left(\mathbf{p}_{4}\right) f_{4}\left(N, \mathbf{p}_{1}, \mathbf{p}_{2}, \mathbf{p}_{3}, \mathbf{p}_{4}\right) \\
+ & \left.\int d \mathbf{p}_{\mathbf{1}} d \mathbf{p}_{\mathbf{2}} d \mathbf{p}_{\mathbf{3}} d \mathbf{p}_{4} d \mathbf{p}_{\mathbf{5}} y\left(\mathbf{p}_{\mathbf{1}}\right) y\left(\mathbf{p}_{\mathbf{2}}\right) y\left(\mathbf{p}_{\mathbf{3}}\right) y\left(\mathbf{p}_{4}\right) y\left(\mathbf{p}_{\mathbf{5}}\right) f_{5}\left(N, \mathbf{p}_{\mathbf{1}}, \mathbf{p}_{\mathbf{2}}, \mathbf{p}_{\mathbf{3}}, \mathbf{p}_{4}, \mathbf{p}_{\mathbf{5}}\right)\right]
\end{aligned}
$$

The $s$ particles inclusive distribution can be written down as

$$
\rho\left(\mathbf{p}_{\mathbf{1}}, \mathbf{p}_{\mathbf{2}}, \ldots, \mathbf{p}_{\mathbf{s}}\right)=\sum_{N=0}^{\infty} f_{s}\left(N, \mathbf{p}_{\mathbf{1}}, \mathbf{p}_{\mathbf{2}}, \ldots, \mathbf{p}_{\mathbf{s}}\right)
$$

with

$$
\int d \mathbf{p}_{\mathbf{1}} \ldots d \mathbf{p}_{\mathbf{s}} \rho\left(\mathbf{p}_{\mathbf{1}}, \ldots, \mathbf{p}_{\mathbf{s}}\right)=\sum_{N=0}^{\infty} P(N) \frac{N !}{(N-s) !}=\langle N(N-1) \ldots(N-s+1)\rangle .
$$

Then we have [20]:

$$
\begin{gathered}
\langle Z(y)\rangle=\int y(\mathbf{p}) \rho(\mathbf{p}) d \mathbf{p} \\
\left\langle Z(y)^{2}\right\rangle=\int y^{2}(\mathbf{p}) \rho(\mathbf{p}) d \mathbf{p}+\int d \mathbf{p}_{\mathbf{1}} d \mathbf{p}_{\mathbf{2}} y\left(\mathbf{p}_{\mathbf{1}}\right) y\left(\mathbf{p}_{\mathbf{2}}\right) \rho\left(\mathbf{p}_{\mathbf{1}}, \mathbf{p}_{\mathbf{2}}\right) \\
\left\langle Z(y)^{3}\right\rangle=\int y^{3}(\mathbf{p}) \rho(\mathbf{p}) d \mathbf{p} \\
+3 \int d \mathbf{p}_{\mathbf{1}} d \mathbf{p}_{\mathbf{2}} y^{2}\left(\mathbf{p}_{\mathbf{1}}\right) y\left(\mathbf{p}_{\mathbf{2}}\right) \rho\left(\mathbf{p}_{\mathbf{1}}, \mathbf{p}_{\mathbf{2}}\right) \\
+\int d \mathbf{p}_{\mathbf{1}} d \mathbf{p}_{\mathbf{2}} d \mathbf{p}_{\mathbf{3}} y\left(\mathbf{p}_{\mathbf{1}}\right) y\left(\mathbf{p}_{\mathbf{2}}\right) y\left(\mathbf{p}_{\mathbf{3}}\right) \rho\left(\mathbf{p}_{\mathbf{1}}, \mathbf{p}_{\mathbf{2}}, \mathbf{p}_{\mathbf{3}}\right)
\end{gathered}
$$




$$
\begin{aligned}
& \left\langle Z(y)^{4}\right\rangle=\int y^{4}(\mathbf{p}) \rho(\mathbf{p}) d \mathbf{p}+4 \int d \mathbf{p}_{\mathbf{1}} d \mathbf{p}_{\mathbf{2}} y^{3}\left(\mathbf{p}_{\mathbf{1}}\right) y\left(\mathbf{p}_{\mathbf{2}}\right) \\
& +3 \int d \mathbf{p}_{\mathbf{1}} d \mathbf{p}_{\mathbf{2}} y^{2}\left(\mathbf{p}_{\mathbf{1}}\right) y^{2}\left(\mathbf{p}_{\mathbf{2}}\right) \rho\left(\mathbf{p}_{\mathbf{1}}, \mathbf{p}_{\mathbf{2}}\right) \\
& +6 \int d \mathbf{p}_{\mathbf{1}} d \mathbf{p}_{\mathbf{2}} d \mathbf{p}_{\mathbf{3}} y^{2}\left(\mathbf{p}_{\mathbf{1}}\right) y\left(\mathbf{p}_{\mathbf{2}}\right) y\left(\mathbf{p}_{\mathbf{3}}\right) \rho\left(\mathbf{p}_{\mathbf{1}}, \mathbf{p}_{\mathbf{2}}, \mathbf{p}_{\mathbf{3}}\right) \\
& +\int d \mathbf{p}_{\mathbf{1}} d \mathbf{p}_{\mathbf{2}} d \mathbf{p}_{\mathbf{3}} d \mathbf{p}_{\mathbf{4}} y\left(\mathbf{p}_{\mathbf{1}}\right) y\left(\mathbf{p}_{\mathbf{2}}\right) y\left(\mathbf{p}_{\mathbf{3}}\right) y\left(\mathbf{p}_{\mathbf{4}}\right) \rho\left(\mathbf{p}_{\mathbf{1}}, \mathbf{p}_{\mathbf{2}}, \mathbf{p}_{\mathbf{3}}, \mathbf{p}_{\mathbf{4}}\right),
\end{aligned}
$$

and so on. One can use Eq.(4) or Eqs(18, 19, 20) to calculate $\left\langle Z(y)^{K}\right\rangle$ separately to examine the consistency between those equations. The discussions about the consistency between those equations can be found in Ref. [20].

\section{III. $\Phi$-MEASURE OF PIONS WITH MULTI-PARTICLES BOSE-EINSTEIN SYMMETRIZATION CORRELATIONS}

In the following we will only consider pions $\left(\pi^{+}\right.$or $\left.\pi^{-}\right)$, the abundant particles produced in high energy collisions. The $N$ pions distribution function can be written down as 23]

$$
f\left(N, \mathbf{p}_{\mathbf{1}}, \ldots, \mathbf{p}_{\mathbf{N}}\right)=P(N) \frac{P_{N}\left(\mathbf{p}_{\mathbf{1}}, \mathbf{p}_{\mathbf{2}}, \ldots, \mathbf{p}_{\mathbf{N}}\right)}{\int d \mathbf{p}_{\mathbf{1}} \ldots d \mathbf{p}_{\mathbf{N}} P_{N}\left(\mathbf{p}_{\mathbf{1}}, \ldots, \mathbf{p}_{\mathbf{N}}\right)}
$$

with

$$
P_{N}\left(\mathbf{p}_{\mathbf{1}}, \ldots, \mathbf{p}_{\mathbf{N}}\right)=\sum_{\sigma} \rho^{(N)}\left(\mathbf{p}_{\mathbf{1}}, \mathbf{p}_{\sigma(\mathbf{1})}\right) \ldots \rho^{(N)}\left(\mathbf{p}_{\mathbf{N}}, \mathbf{p}_{\sigma(\mathbf{N})}\right)
$$

Here $\rho^{(N)}\left(\mathbf{p}_{\mathbf{i}}, \mathbf{p}_{\mathbf{j}}\right)$ is the Fourier transformation of the pion source distribution function $g^{(N)}(\mathbf{x}, \mathbf{K})$, and $g^{(N)}(\mathbf{x}, \mathbf{K})$ is the probability of finding a pion at point $\mathbf{x}$ with momentum $\mathbf{K}$. The superscript $N$ denotes those quantities are calculated for a system with pion multiplicity $N . \sigma(i)$ denotes the $i$-th element of a permutations of the sequence $\{1,2, \cdots, s\}$, and the sum over $\sigma$ denotes the sum over all $s$ ! permutations of this sequence.

Using Eqs.(6, 21, 22, , we have [24,25]:

$$
\begin{gathered}
f_{s}\left(N, \mathbf{p}_{\mathbf{1}}, \cdots \mathbf{p}_{\mathbf{s}}\right)=\frac{P(N)}{\omega^{(N)}(N)} \sum_{i=s}^{N} \sum_{m_{1}=1}^{i-(s-1)} \sum_{m_{2}=1}^{i-m_{1}-(s-2)} \cdots \\
\sum_{m_{s-1}=1}^{i-m_{1}-m_{2} \cdots-m_{s-2}-1} \sum_{\sigma} G_{m_{1}}^{(N)}\left(\mathbf{p}_{1}, \mathbf{p}_{\sigma(1)}\right) G_{m_{2}}^{(N)}\left(\mathbf{p}_{2}, \mathbf{p}_{\sigma(2)}\right) \\
\cdots G_{m_{s-1}}^{(N)}\left(\mathbf{p}_{s-1}, \mathbf{p}_{\sigma(s-1)}\right) G_{i-m_{1} \cdots-m_{s-1}}^{(N)}\left(\mathbf{p}_{s}, \mathbf{p}_{\sigma(s)}\right) \omega^{(N)}(N-i)
\end{gathered}
$$

with

$$
\begin{aligned}
& \omega^{(N)}(N)=\frac{1}{N !} \int d \mathbf{p}_{\mathbf{1}} \ldots d \mathbf{p}_{\mathbf{N}} P_{N}\left(\mathbf{p}_{\mathbf{1}}, \ldots, \mathbf{p}_{\mathbf{N}}\right) \\
& =\frac{1}{N} \sum_{i=1}^{N} \omega^{(N)}(N-i) \int d \mathbf{p} G_{i}^{(N)}(\mathbf{p}, \mathbf{p}), \\
& \omega^{(N)}(0)=1, G_{1}^{N}=\rho^{N}(\mathbf{p}, \mathbf{p})
\end{aligned}
$$


and

$$
G_{i}^{(N)}(\mathbf{p}, \mathbf{q})=\int \rho^{(N)}\left(\mathbf{p}, \mathbf{p}_{\mathbf{1}}\right) d \mathbf{p}_{\mathbf{1}} \rho^{(N)}\left(\mathbf{p}_{\mathbf{1}}, \mathbf{p}_{\mathbf{2}}\right) \cdots d \mathbf{p}_{\mathbf{i}-\mathbf{1}} \rho^{(N)}\left(\mathbf{p}_{\mathbf{i}-\mathbf{1}}, \mathbf{q}\right)
$$

The superscript $N$ of $\omega^{(N)}$ and $G_{i}^{(N)}(\mathbf{p}, \mathbf{q})$ denote $\omega^{(N)}$ and $G_{i}^{(N)}(\mathbf{p}, \mathbf{q})$ are calculated using the quantities $\rho^{(N)}\left(\mathbf{p}_{\mathbf{i}}, \mathbf{p}_{\mathbf{j}}\right)$.

The $s$-pion inclusive distribution can be expressed as

$$
\begin{aligned}
& \rho\left(\mathbf{p}_{\mathbf{1}}, \cdots, \mathbf{p}_{\mathbf{s}}\right)=\sum_{N=0}^{\infty} f_{s}\left(N, \mathbf{p}_{\mathbf{1}}, \ldots, \mathbf{p}_{\mathbf{s}}\right) \\
& =\sum_{N=s}^{\infty} \sum_{i=s}^{N} \sum_{m_{1}=1}^{i-(s-1)} \sum_{m_{2}=1}^{i-m_{1}-(s-2)} \ldots \sum_{m_{s-1}=1}^{i-m_{1}-m_{2} \cdots-m_{s-2}-1} P(N) \frac{\omega^{N}(N-i)}{\omega^{N}(N)} \sum_{\sigma} \\
& G_{m_{1}}^{(N)}\left(\mathbf{p}_{1}, \mathbf{p}_{\sigma(1)}\right) G_{m_{2}}^{(N)}\left(\mathbf{p}_{2}, \mathbf{p}_{\sigma(2)}\right) \cdots G_{m_{s-1}}^{(N)}\left(\mathbf{p}_{s-1}, \mathbf{p}_{\sigma(s-1)}\right) G_{i-m_{1} \cdots-m_{s-1}}^{(N)}\left(\mathbf{p}_{s}, \mathbf{p}_{\sigma(s)}\right) \\
& =\sum_{m_{1}=1}^{\infty} \cdots \sum_{m_{s}=1}^{\infty} \sum_{N=m_{1}+m_{2}+\ldots+m_{s}}^{\infty} P(N) \frac{\omega^{(N)}\left(N-m_{1}-m_{2}-\ldots-m_{s}\right)}{\omega^{(N)}(N)} \\
& \sum_{\sigma} G_{m_{1}}^{(N)}\left(\mathbf{p}_{1}, \mathbf{p}_{\sigma(1)}\right) \cdots G_{m_{s}}^{(N)}\left(\mathbf{p}_{s}, \mathbf{p}_{\sigma(s)}\right) .
\end{aligned}
$$

Neglecting symmetrization effects, that is $\rho^{(N)}\left(p_{i}, p_{j}\right)=0$, we have

$$
\omega^{(N)}(N)=\frac{1}{N !}
$$

and

$$
G_{k}^{(N)}\left(\mathbf{p}_{\mathbf{i}}, \mathbf{p}_{\mathbf{j}}\right)=0, \quad k \neq 1 \cup \mathbf{p}_{\mathbf{i}} \neq \mathbf{p}_{\mathbf{j}}
$$

Thus Eq.(23) reads

$$
\begin{aligned}
f_{s}\left(N, \mathbf{p}_{\mathbf{1}}, \ldots, \mathbf{p}_{\mathbf{s}}\right) & =\frac{P(N)}{\omega^{(N)}(N)} G_{1}^{N}\left(\mathbf{p}_{\mathbf{1}}, \mathbf{p}_{\mathbf{1}}\right) \ldots G_{1}^{N}\left(\mathbf{p}_{\mathbf{s}}, \mathbf{p}_{\mathbf{s}}\right) \omega^{(N)}(N-s) \\
& =P(N) \frac{N !}{(N-s) !} \rho^{N}\left(\mathbf{p}_{\mathbf{1}}, \mathbf{p}_{\mathbf{1}}\right) \ldots \rho^{N}\left(\mathbf{p}_{\mathbf{s}}, \mathbf{p}_{\mathbf{s}}\right) .
\end{aligned}
$$

From Eq.(15) or Eq.(26) we have

$$
\rho\left(\mathbf{p}_{\mathbf{1}}, \ldots, \mathbf{p}_{\mathbf{s}}\right)=\sum_{N=s}^{\infty} N(N-1) \ldots(N-s+1) P(N) \rho^{N}\left(\mathbf{p}_{\mathbf{1}}, \mathbf{p}_{\mathbf{1}}\right) \ldots \rho^{N}\left(\mathbf{p}_{\mathbf{s}}, \mathbf{p}_{\mathbf{s}}\right) .
$$

If we neglect multi-particle symmetrization(or antisymmetrization) effects and the correlation between particle spectrum distribution and multiplicity, we immediately come to the conclusion that $\rho^{N}(\mathbf{p}, \mathbf{p})=\rho(\mathbf{p}) /\langle N\rangle$. Of course this conclusion is based on the fact that we have neglected the dynamical correlation and kinematic correlation.

In calculation, one normally choose the variable $y(\mathbf{p})$ as [9]

$$
y(\mathbf{p})=x(\mathbf{p})-\bar{x}, \quad \bar{x}=\int d \mathbf{p} x(\mathbf{p}) \frac{\rho(\mathbf{p})}{\langle N\rangle} .
$$


If we neglect all kinds of correlations in the system, the first three moments of $Z$ read:

$$
\left\langle Z^{k}\right\rangle=\int d \mathbf{p} y^{k}(\mathbf{p}) \rho(\mathbf{p}) \quad(1 \leq k \leq 3) .
$$

For high-order moments, Eq.(32) does not hold anymore. This can be easily seen from Eqs.(13), 14, 20). This result is different from Ref. [21] where Eq.(32) have been thought to be valid for all-order moments. Mrowczynski has also noticed this fact in Ref. [17]. So $\Phi$-measure

$$
\Phi_{k}=\left(\frac{\left\langle Z^{k}\right\rangle}{\langle N\rangle}\right)^{1 / k}-\left(\overline{y^{k}}\right)^{1 / k} \quad(2 \leq k \leq 3)
$$

with

$$
\overline{y^{k}}=\frac{1}{\langle N\rangle} \int \rho(\mathbf{p}) y^{k}(\mathbf{p}) d \mathbf{p},
$$

is related to the quantum many-body correlations, the correlation between the multiplicity and momentum distribution, and the dynamical correlations, the last one has already been discussed in Ref. [9].

As we have no interest in the first two kinds of correlations, we will eliminate those correlations from the $\Phi$-measure. One may get rid of multiplicity and momentum correlation by redefining the variable $y(\mathbf{p})$ as

$$
y^{(N)}(\mathbf{p})=x(\mathbf{p})-\overline{x^{(N)}}
$$

with

$$
\overline{x^{(N)}}=\int d \mathbf{p} x(\mathbf{p}) \frac{f_{1}(N, \mathbf{p})}{N} .
$$

Here $\frac{f_{1}(N, \mathbf{p})}{N}$ is the normalized particle spectrum distribution of pions with multiplicity $N$. After this definition one may get rid of the possible correlation between the multiplicity and momentum. In the actual calculation, one may calculate the average value of $x(\mathbf{p})$ for each event(denotes as $\left.x^{e}\right)$, then uses the redefined $y(\mathbf{p})=x(\mathbf{p})-x^{e}$ and Eq.(4) to calculate $\left\langle Z^{k}\right\rangle$. For the $\overline{y^{k}}$, one may calculate it using following equation

$$
\overline{y^{k}}=\frac{1}{M} \sum_{j=1}^{M} \sum_{i=1}^{N_{j}} y_{j}\left(\mathbf{p}_{\mathbf{i}}\right)^{k}
$$

Here $M$ is the total number of events and $N_{j}$ is the multiplicity of the $j$-th event. $y_{j}(\mathbf{p})=$ $x(\mathbf{p})-x_{j}^{e}$ is the quantity defined for the $j$-th event, and $x_{j}^{e}$ is mean value of $x(\mathbf{p})$ for the $j$-th event. Using this method, one can calculate the $\Phi$-measure.

In the following we will calculate the quantum correlation effects on $\Phi$-measure. Assuming $g^{(N)}(\mathbf{x}, \mathbf{K})$ as a Gaussian function in both coordinate space and momentum space, $\rho^{(N)}\left(\mathbf{p}_{\mathbf{i}}, \mathbf{p}_{\mathbf{j}}\right)$ can be expressed as 24,25

$$
\begin{aligned}
\rho^{(N)}\left(\mathbf{p}_{\mathbf{i}}, \mathbf{p}_{\mathbf{j}}\right) & =\int g\left(\mathbf{x}, \frac{\mathbf{p}_{\mathbf{i}}+\mathbf{p}_{\mathbf{j}}}{2}\right) \exp \left(i\left(\mathbf{p}_{\mathbf{i}}-\mathbf{p}_{\mathbf{j}}\right) \cdot x\right) d \mathbf{x} \\
& =\frac{1}{\left(2 \pi \Delta^{2}\right)^{\frac{3}{2}}} \exp \left(-\frac{\left(\mathbf{p}_{\mathbf{i}}-\mathbf{p}_{\mathbf{j}}\right)^{2} R(N)^{2}}{2}\right) \exp \left(-\frac{\left(\mathbf{p}_{\mathbf{i}}+\mathbf{p}_{\mathbf{j}}\right)^{2}}{8 \Delta(N)^{2}}\right) .
\end{aligned}
$$


Here $R(N)$ and $\Delta(N)$ are the source radius and temperature for the events with multiplicity $N$. Of course the source radius and temperature may be different for different event, even though those events have the same multiplicity. But at this calculation we will neglect this fluctuation. In Fig.1, $\Phi_{2}\left(p_{t}\right)$ vs. mean pion multiplicity are shown ? It is interesting to note that $\Phi$ value will increase as the pion multiplicity goes up. For the same multiplicity, $\Phi$ value will decrease as the volume or temperature drops. All these can be understood in the following way: $\Phi_{2}\left(p_{t}\right)$ will become bigger as the phase space density $(\sim<N>$ $\left./(R \Delta)^{3}\right)$ becomes higher. Of course, the $\Phi$ value should also depend on the pion multiplicity distribution [24,225,27], but this effect will be neglected at present calculation. Here we would like to point out that many authors have noticed that Bose-Einstein symmetrization may affect the value of $\Phi$-measure. For example, In Ref. [21], similar formulas as Eqs (10, 11, 12) of the paper have been derived, but the functional form of $\rho\left(\mathbf{p}_{\mathbf{1}}, \ldots, \mathbf{p}_{\mathbf{s}}\right)$ have never been presented. On the other hand, Mrowczynski has studied Bose-Einstein correlation effects on the $\Phi$-measure by assuming the particle spectrum distribution is Bose-Einstein form

${ }^{3}$ Here, we will explain how to calculate the $\Phi$-measure given in Fig.1. According to Eq. (33,34), we have

$$
\Phi_{2}\left(p_{t}\right)=\left(\frac{\left\langle Z^{2}\right\rangle}{\langle N\rangle}\right)^{1 / 2}-\left(\frac{1}{\langle N\rangle} \int \rho(\mathbf{p}) \tilde{p}_{t}^{2} d \mathbf{p}\right)^{1 / 2}
$$

with

$$
\tilde{p}_{t}=p_{t}-\left\langle p_{t}\right\rangle,\left\langle p_{t}\right\rangle=\frac{1}{\langle N\rangle} \int \rho(\mathbf{p}) p_{t}
$$

Here $p_{t}$ is transverse momentum $\left(p_{t}=\sqrt{p_{1}^{2}+p_{2}^{2}}\right),\langle N\rangle$ is the mean multiplicity. According to Eq.(18), we have

$$
\left\langle Z^{2}\left(p_{t}\right)\right\rangle=\int \tilde{p}_{t}^{2} \rho(p) d \mathbf{p}+\int d \mathbf{p}_{\mathbf{1}} d \mathbf{p}_{\mathbf{2}} \tilde{p}_{1 t} \tilde{p}_{2 t} \rho\left(\mathbf{p}_{\mathbf{1}}, \mathbf{p}_{\mathbf{2}}\right) .
$$

According to Eq.(26), the single and two-particles inclusive distribution can be written down as

$$
\begin{gathered}
\rho(\mathbf{p})=\sum_{m_{1}=1}^{\infty} \sum_{N=m_{1}}^{\infty} P(N) \frac{\omega\left(N-m_{1}\right)}{\omega(N)} G_{m_{1}}(\mathbf{p}, \mathbf{p}), \\
\rho\left(\mathbf{p}_{\mathbf{1}}, \mathbf{p}_{\mathbf{2}}\right)=\sum_{m_{1}=1}^{\infty} \sum_{m_{1}=2}^{\infty} \sum_{N=m_{1}+m_{2}}^{\infty} P(N) \frac{\omega\left(N-m_{1}-m_{2}\right)}{\omega(N)} \\
{\left[G_{m_{1}}\left(\mathbf{p}_{\mathbf{1}}, \mathbf{p}_{\mathbf{2}}\right) G_{m_{2}}\left(\mathbf{p}_{\mathbf{2}}, \mathbf{p}_{\mathbf{1}}\right)+G_{m_{1}}\left(\mathbf{p}_{\mathbf{1}}, \mathbf{p}_{\mathbf{1}}\right) G_{m_{2}}\left(\mathbf{p}_{\mathbf{2}}, \mathbf{p}_{\mathbf{2}}\right)\right] .}
\end{gathered}
$$

In the calculation we assume that $R$ and $\Delta$ are the same for all multiplicity, so we neglect the superscript $N$ in Eq. 26). From the function $\rho^{(N)}\left(p_{i}, p_{j}\right)$ given in Eq.(38), one can calculate $G_{m_{1}}\left(p_{1}, p_{2}\right)$ according to Eq.(25) and $\omega(N)$ according to Eq.(24). Choosing $P(N)$ as Poisson form, that is $P(N)=\exp (-\langle N\rangle) \frac{\langle N\rangle^{N}}{N !}$, we can calculate $\rho\left(\mathbf{p}_{\mathbf{1}}, \mathbf{p}_{\mathbf{2}}\right)$ and $\rho(\mathbf{p})$. Bring all those formulas in eq.(39), we get $\Phi_{2}\left(p_{t}\right)$. 
which corresponds to $R$ is infinity for our calculation [23]. However Neither Belkacem et. al. nor Mrowczynski has calculated multi-body Bose-Einstein symmetrization effects on $\Phi$-measure as done in this paper. For comparison the results of Ref. [12] are also shown in Fig.1 1 . The specific volume $\left(V=L^{3}=1000 \mathrm{fm}^{3}\right)$ and temperature $(T=150 \mathrm{MeV})$ are chosen for the formulas of Ref. [12] so that $\left\langle r^{2}=x^{2}+y^{2}+z^{2}\right\rangle_{\text {box }, L=10 \mathrm{fm}} \sim\left\langle r^{2}\right\rangle_{\text {Gaussian, } R=3 \mathrm{fm}}$ and $\left\langle p^{2}\right\rangle_{T=150 \mathrm{MeV}} \sim\left\langle p^{2}\right\rangle_{\Delta_{0}=0.2 \mathrm{GeV}}$. It is interesting to notice that our results is larger than the results of Ref. [12] when the phase space $\left(\sim\left(\left\langle r^{2}\right\rangle\left\langle p^{2}\right\rangle\right)^{3 / 2}\right)$ are the same. $\Phi_{2}\left(p_{t}\right)$ for Ref. 12 will also become bigger if the phase space density becomes higher. Here we should point out that for $\langle N\rangle \sim 100$, which is the mean multiplicity of $\pi^{+}$or $\pi^{-}$measured in SPS energy, our $\Phi\left(p_{t}\right)$ is much larger than the experimental value of $4.6 \pm 1.5$ 19. However for large volume $(R=5.3 \mathrm{fm}$ and $\Delta=0.2 \mathrm{GeV})$ or high temperature $(R=3 \mathrm{fm}$ and $\Delta=0.355 \mathrm{GeV})$, we find that $\Phi \sim 7 \mathrm{MeV}$ for $<N>\sim 100$ which is consistent with the $\Phi$ value calculated by two-particles correlation $(5 \pm 1.5 \mathrm{MeV})$ for $\mathrm{Pb}+\mathrm{Pb}$ at SPS [19]. Taking $V=L^{3}=(16)^{3} \mathrm{fm}^{3}$ and $T=150 \mathrm{MeV}$, we find that $\Phi$ value of Ref. [12] is around $6 \mathrm{MeV}$ which is consistent with our results and data. This suggests that for large volume $(R=5.1 \mathrm{fm}$ or $L=16 \mathrm{fm}$ which is around the HBT radius of NA49 [28]), high-order Bose-Einstein symmetrization effects are not significant for SPS energy. So our calculation is consistent with the results of Ref. [12].

\footnotetext{
${ }^{4}$ Here we will explain how to calculate the $\Phi$-measure using the method given in Ref. [12]. Ac-
} cording to Ref. [12], we can infer that

$$
\rho(\mathbf{p})=\frac{V}{(2 \pi)^{3}} \frac{1}{\exp (\beta(E-\mu))-1} .
$$

Here $V=L^{3}$ is the volume, $\beta=T^{-1}$ is the inverse temperature, $\mu$ is the chemical potential. $E=\sqrt{m^{2}+p^{2}}$ with $m$ being the particles mass and $\mathbf{p}$ its momentum. The mean multiplicity $\langle N\rangle$ can be expressed as

$$
\langle N\rangle=\int d \mathbf{p} \rho(\mathbf{p})
$$

The mean $\left\langle p_{t}\right\rangle$ reads

$$
\left\langle p_{t}\right\rangle=\frac{\int \rho(\mathbf{p}) p_{t} d \mathbf{p}}{\langle N\rangle}
$$

It is easily checked that [12]

$$
\frac{\left\langle Z^{2}\left(p_{t}\right)\right\rangle}{\langle N\rangle}=\frac{\int d \mathbf{p}\left(p_{t}-\left\langle p_{t}\right\rangle\right)^{2} \frac{\exp (\beta(E-\mu))}{(\exp (\beta(E-\mu))-1)^{2}}}{\int d \mathbf{p} \frac{1}{\exp (E-\mu)-1}} .
$$

Bring those formulas in Eq.(39), we can get $\Phi_{2}\left(p_{t}\right)$. 


\section{THE SHORTCOMING OF PREVIOUS DERIVATION OF $\Phi$-MEASURE}

In the following we show that the previous derivation of the $\Phi$-measure is based on a model which has no quantum correlation among particles. We assume that in AA collisions, particles are produced by each nucleon nucleon $(N N)$ collisions and the number of collisions of NN has a distribution function $P(M)$. Here $M$ is the number of collisions in each event and the collision center has a distribution of $\rho(\mathbf{x})$. The probability amplitude of finding a pion at point $\mathbf{x}$ can be written down as 29

$$
J(\mathbf{x})=\sum_{i=1}^{M} J_{i}(\mathbf{x})=\sum_{i=1}^{M} J\left(\mathbf{x}-\mathbf{x}_{\mathbf{i}}\right)
$$

Here $\mathbf{x}_{\mathbf{i}}$ is the collision center coordinate. In the last step of Eq. (48) we assume that $J_{i}(\mathbf{x})$ is only the function of $\mathbf{x}-\mathbf{x}_{\mathbf{i}}$. The probability amplitude in the momentum space can be expressed as 29]

$$
\tilde{J}(\mathbf{p})=\int d \mathbf{x} J(\mathbf{x}) \exp (i \mathbf{p} \cdot \mathbf{x})=\sum_{i=1}^{M} \int \exp (i \mathbf{p} \cdot \mathbf{x}) J\left(\mathbf{x}-\mathbf{x}_{\mathbf{i}}\right) d \mathbf{x}=\sum_{i=1}^{M} \tilde{J}(\mathbf{p}) \exp \left(i \mathbf{p} \cdot \mathbf{x}_{\mathbf{i}}\right)
$$

with

$$
\tilde{J}(\mathbf{p})=\int J(\mathbf{x}) \exp (i \mathbf{p} \cdot \mathbf{x}) d \mathbf{x}
$$

Then the $s$-pion inclusive distribution reads

$$
\begin{gathered}
\rho\left(\mathbf{p}_{\mathbf{1}}, \ldots, \mathbf{p}_{\mathbf{s}}\right)=\sum_{M=0}^{\infty} P(M) \int d \mathbf{x}_{\mathbf{1}} \ldots d \mathbf{x}_{\mathbf{M}} g\left(\mathbf{x}_{\mathbf{1}}\right) \ldots g\left(\mathbf{x}_{\mathbf{M}}\right)\left|\widetilde{J}\left(\mathbf{p}_{\mathbf{1}}\right)\right|^{2} \ldots\left|\widetilde{J}\left(\mathbf{p}_{\mathbf{s}}\right)\right|^{2} \\
\left|\sum_{i=1}^{M} \exp \left(i \mathbf{p}_{\mathbf{1}} \cdot \mathbf{x}_{\mathbf{i}}\right)\right|^{2} \ldots\left|\sum_{i=1}^{M} \exp \left(i \mathbf{p}_{\mathbf{s}} \cdot \mathbf{x}_{\mathbf{i}}\right)\right|^{2}
\end{gathered}
$$

the second line of Eq.(51) comes from Eq.(49) which is due to quantum effect. So the distribution $P(X)$ in Ref. [17] does not hold anymore. However if we consider classical particles, we immediately can get an equation similar to the expression of $P(X)$ in Ref. [17]. As the terms in the second line of Eq.(51) change to

$$
\left|\sum_{i=1}^{M} \exp \left(i \mathbf{p}_{\mathbf{j}} \cdot \mathbf{x}_{\mathbf{i}}\right)\right|^{2} \rightarrow \sum_{i=1}^{M}\left|\exp \left(i \mathbf{p}_{\mathbf{j}} \cdot \mathbf{x}_{\mathbf{i}}\right)\right|^{2}=M
$$

we have

$$
\rho\left(\mathbf{p}_{\mathbf{1}}, \mathbf{p}_{\mathbf{2}}, \ldots, \mathbf{p}_{\mathbf{s}}\right)=\sum_{M=0}^{M=\infty} P(M) M^{s}\left|\widetilde{J}\left(\mathbf{p}_{\mathbf{1}}\right)\right|^{2} \ldots\left|\widetilde{J}\left(\mathbf{p}_{\mathbf{s}}\right)\right|^{2}
$$

Then the derivation in Ref [17] can be applied here. In passing, we would like to note that the $P(X)$ distribution in Ref. [17] is the source distribution, while, $\rho\left(\mathbf{p}_{\mathbf{1}}, \ldots, \mathbf{p}_{\mathbf{s}}\right)$ in our work represents particle inclusive distribution. For illustration, we will write out the mean multiplicity $\langle N\rangle$ as: 


$$
\begin{aligned}
\langle N\rangle & =\int \rho\left(\mathbf{p}_{\mathbf{1}}\right) d \mathbf{p}_{\mathbf{1}} \\
& =\langle M\rangle \int d \mathbf{p}_{\mathbf{1}}\left|J\left(\mathbf{p}_{\mathbf{1}}\right)\right|^{2}+\langle M(M-1)\rangle \int d \mathbf{p}_{\mathbf{1}} \mid J\left(\left.\mathbf{p}_{\mathbf{1}}\right|^{2}\left|\widetilde{g}\left(\mathbf{p}_{\mathbf{1}}\right)\right|^{2}\right.
\end{aligned}
$$

with

$$
\widetilde{g}\left(\mathbf{p}_{\mathbf{1}}\right)=\int d \mathbf{x} g(\mathbf{x}) \exp \left(i \mathbf{p}_{\mathbf{1}} \cdot \mathbf{x}\right)
$$

Here $\int d \mathbf{p}_{\mathbf{1}}\left|J\left(\mathbf{p}_{\mathbf{1}}\right)\right|^{2}=n_{0}$ is the mean number of pions that are emitted from each NN collisions. The previous relation like $\langle N\rangle=\langle M\rangle n_{0}$ does not hold anymore as the quantum effect has extra contribution to the average value of $\langle N\rangle$. Of course the last term of Eq. (54) depends on the distribution function of the source, $g(\mathbf{x})$, and it will become smaller if the collision region is larger. This can be explained in quantum mechanical context: If the source of AA collisions is very larger and dilute pion gas exists, quantum effect will become smaller.

\section{DISCUSSIONS AND CONCLUSIONS}

It has been proved that $\Phi$-measure will be significantly influenced by the multiparticle correlations effects if the particle phase space density is very high. However one may exclude the quantum symmetrization or antisymmetrization effects by cuts. One may do HBT analysis and determine the HBT momentum region first, then throw away the identical particle pairs which are affected greatly by particles symmetrization effects from each event. After this is done one may use the remaining particles to re-calculate the $\Phi$ measure. It is interesting to mention the following fact: In Ref. [30,31], the authors have already calculated the average phase space density for pions at SPS energy, and have found that the pion phase space density is 0.2 , which is much less than 1 . Therefore multipion symmetrization effect may not be significant at SPS energy. For RHIC energy, it is estimated that the pion phase space density will become higher, so one should be careful when using $\Phi$-measure in RHIC energy.

In the paper, we calculate the $\Phi$-measure for a specific source distribution function. There is a possibility that the results of $\Phi$-measure depends on the function form of the source. As has been stated in the paper that $\Phi$-measure also depend on the fluctuation of the source radius and temperature for different multiplicity. All this kind of work has not been studied in detail in the paper as the main interest of present paper is to study the Bose-Einstein correlation effects on the $\Phi$-measure. We find that $\Phi$-measure is greatly affected by Bose-Einstein symmetrization if the source density of pions is high.

In this paper, we derive the relationship between $\Phi$-measure and particles inclusive distributions. It is shown that besides dynamical correlation, $\Phi$-measure is also related to the many-body quantum (anti)symmetrization correlations. Starting from a pion source distribution in coordinate and momentum space, we calculate the $\Phi$-measure for pions. It is found that $\Phi$-measure is strongly affected by multiparticle symmetrization effects if the phase space density is very high. So the previous statement that $\Phi$-measure reflects the dynamical mechanism that exists in the AA does not hold anymore if the pion phase space density is high. For a very large system, the density of pions may be smaller, in this case 
the multiparticles symmetrization effect can be neglected. Finally we argue that one may more or less exclude the quantum symmetrization effect by cutting small momentum pairs of pions from each event.

\section{Acknowledgement}

The author thanks Prof. R. C. Hwa for reading the paper and Dr. S. Mrowczynski for helpful communication. This work was supported by US Department of Energy under Grant No. DE-FG03-96ER40972, NSERC of Canada and FCAR of the Quebec Government. 


\section{REFERENCES}

[1] Quark Gluon Plasma, edited by R. C. Hwa, (World scientific, Singapore),1990; Quark Gluon Plasma 2, edited by R. C. Hwa, (World scientific, Singapore),1995; U. Heinz and M. Jacob, nucl-th/0002042.

[2] A. Bialas and R. Peschanski, Nucl. Phys. B308,857 (1986); Z. Cao and R. C. Hwa, Phys. Rev. Lett. 75, 1268 (1995); Phys. Rev. D56, 7361 (1997); R. C. Hwa and Q. H. Zhang, Phys. Rev. D 62014003 (2000).

[3] R. C. Hwa and Q. H. Zhang, nucl-th/0104019; Phys. Rev. C62 054902 (2000); R. C. Hwa and C. B. Yang, hep-ph/0104216.

[4] L. Stodolsky, Phys. Rev. Lett. 75, 1044 (1995).

[5] T. A. Trainor, hep-ph/0001148.

[6] M. Bleicher, et al, Nucl. Phys. A638, 391c (1998).

[7] E. Shuryak, Phys. Lett. B423, 9 (1998).

[8] M. Stephanov, K. Rajagopal, and E. Shuryak, Phys. Rev. D60, 114028 (1999).

[9] M. Gazdzicki and S. Mrowczynski, Z. Phys. C54, 127 (1992).

[10] M. Gazdzicki, Eur. Phys. J. C8, 131 (1999).

[11] S. Mrowczynski, Phys. Lett. B459, 13 (1999).

[12] S. Mrowczynski, Phys. Lett. B439, 6 (1998).

[13] F. Liu et. al, Euro. Phys. J. C8, 649 (1999).

[14] S. A. Voloshin, V. Koch and H. G. Ritter; nucl-th/9903060.

[15] M. Bleicher et. al. Phys. Lett. B435, 9 (1998).

[16] M. Gazdzicki, A. Leonidov and G. Roland, Eur. Phys. J. C6, 365 (1999).

[17] S. Mrowczynski, Phys. Lett. B465, 8 (1999)

[18] G. Baym and H. Heiselberg, Phys. Lett. B469, 7 (1999).

[19] G. Roland, Nucl. Phys. A638, 91c (1998); H. Appelshauser et. al, NA49, Phys. Lett. B459, (1999) 679.

[20] A. Bialas and V. Koch, Phys. Lett. B456, 1 (1999).

[21] M. Belkacem et.al, nucl-th/9903017.

[22] Q. H. Zhang and S. Padula, Phys. Rev. C62, 024902 (2000).

[23] W. A. Zajc, Phys. Rev. D 35, 3396 (1987); S. Pratt, Phys. Lett. B301, 159(1993); W.Q. Chao, C.S. Gao and Q. H. Zhang, J. Phys. G21, 847 (1995); Q. H. Zhang, W. Q. Chao and C. S. Gao, Phys. Rev. C52, 2064 (1995); T. Csorgo and J. Zimanyi, Phys. Rev Lett 80, 916(1998); J. Zimanyi and T. Csorgo, Heavy-ion physics 9, 241 ( 1999); A. Bialas and K. Zalewski, Phys. Lett. B436, 153 (1998); R. Lednicky et al, nucl-th/9911055;T. Csorgo, hep-ph/0001233.

[24] Q.H. Zhang, Phys. Rev. C59, 1646(1999); Phys. Rev. C58, 18 (1998).

[25] Q.H. Zhang, in Correlation and fluctuation'98,(World Scientific, Singapore)p 74, edited by T. Csorgo et. al. (hep-ph/9807257).

[26] Q. H. Zhang, P. Scotto and U. Heinz, Phys. Rev. C58, 3757 (1998).

[27] U. Heinz, P. Scotto and Q. H. Zhang, Ann. Phys. 288, 325 (2001).

[28] H. Appelshauser et. al, NA49 Collab. , Eur. Phys. J. C2, (1998) 661.

[29] M. Gyulassy,S. K. Kauffmann and L.W. Wilson, Phys. Rev. C20, 2267 (1979).

[30] D. Ference, U. Heinz, B. Tomasik, U. A. Wiedemann and J. Gramer; Phys. Lett B457, 347 (1999).

[31] D. A. Brown, S. Y. Panitkin and G. Bertsch, nucl-th/0002039 


\section{FIGURES}

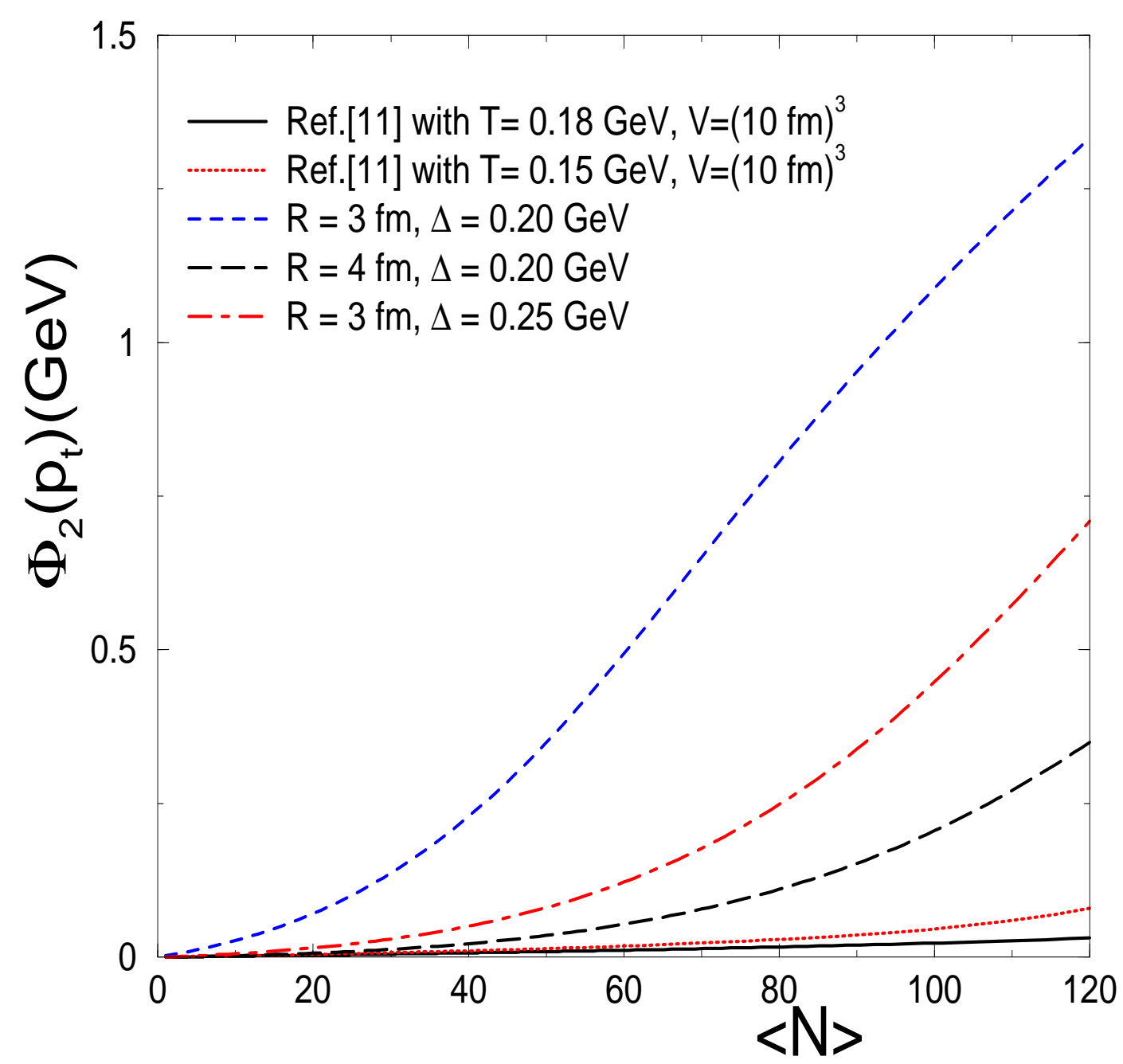

FIG. 1. $\Phi_{2}\left(p_{t}\right)$ vs. mean pion multiplicity $\langle N\rangle$. In the calculation we choose $\Delta=0.2 G e V$, $R=3 \mathrm{fm}$ (dashed line) and $4 \mathrm{fm}$ (long dashed line) respectively. For comparison, $\Phi_{2}\left(p_{t}\right)$ for $\Delta=0.25 \mathrm{GeV}, R=3 \mathrm{fm}$ (dot-dashed line) are also shown in Fig.1. We choose the pion multiplicity distribution, $P(N)$, as Poisson form. The results from Ref.[11] are also shown in Fig.1. We choose the volume of the pion system (box) as $V=L^{3}=1000 \mathrm{fm}^{3}$ and the temperature $T=0.15 G e V$ (dotted line) and $T=0.18 G e V$ (solid line) respectively. 